\title{
Ground Band and a Generalized GP-equation for Spinor Bose-Einstein Condensates
}

\author{
C.G. $\mathrm{Bao}^{1,2}$ and Z.B. $\mathrm{Li}^{1}$ \\ ${ }^{1}$ State Key Laboratory of Optoelectronic Materials and Technologies, \\ and Department of Physics, Zhongshan University, Guangzhou 510275, P.R. China, and \\ ${ }^{2}$ Center of Theoretical Nuclear Physics, National Laboratory of Heavy Ion Accelerator, Lanzhou 780000, P.R. China
}

\begin{abstract}
For the spinor Bose-Einstein condensates both the total spin $S$ and its Z-component $S_{Z}$ should be conserved. However, in existing theories, only the conservation of $S_{z}$ has been taken into account. To remedy, this paper is the first attempt to take the conservation of both $S$ and $S_{Z}$ into account. For this purpose, a total spin-state with the good quantum numbers $S$ and $S_{Z}$ is introduced in the trial wave function, thereby a generalized Gross-Pitaevskii equation has been derived. With this new equation, the ground bands of the ${ }^{23} \mathrm{Na}$ and ${ }^{87} \mathrm{Rb}$ condensates have been studied, where the levels distinct in $S$ split. It was found that the level density is extremely dense in the bottom of the ground band of ${ }^{23} \mathrm{Na}$, i.e., in the vicinity of the ground state. On the contrary, for ${ }^{87} \mathrm{Rb}$, the levels are extremely dense in the top of the ground band,

PACS numbers: 03.75. Fi, 03.65. Fd
\end{abstract}

\section{1 , Introduction}

In 1998 the spinor Bose-Einstein condensation of sodium atoms was experimentally realized in an optical trap by Stamper-Kurn et al [1]. The advantage of the optical trap is that it liberates the spin degrees of freedom of atoms (e.g., ${ }^{23} \mathrm{Na},{ }^{39} \mathrm{~K}$, and ${ }^{87} \mathrm{Rb}$ atoms have nuclear spin $3 / 2$ and electrons at $s$ orbits, they behave as simple bosons with spin one at low temperature). As a consequence of the liberation, such a macroscopically coherent system with internal degrees of freedom becomes very rich in physics. Several interesting phenomena have already been obtained, e.g., quantum entanglement of spins [2, 3], spinor four-wave mixing [4, 5], and super fragmentation and coherent fragmentation $[\underline{6}$.

For the spinor Bose-Einstein condensates of bosons with spin one, the pairwise interaction $U_{i j}$ is spindependent. It can be written as $\mathbf{7},[\underline{8},[9,10,11]$

$$
\begin{aligned}
U_{i j} & =\delta\left(\mathbf{r}_{i}-\mathbf{r}_{j}\right)\left(g_{m} P_{m}+g_{q} P_{q}\right) \\
& =\delta\left(\mathbf{r}_{i}-\mathbf{r}_{j}\right)\left(c_{0}+c_{2} \mathbf{F}_{i} \cdot \mathbf{F}_{j}\right)
\end{aligned}
$$

where $P_{m}$ and $P_{q}$ are the projectors to two-body spinstates with two-body spin equal to 0 and 2, respectively, and $g_{m}$ and $g_{q}$ are the associated strengths. $\mathbf{F}_{i}$ is the spin operator of particle $i, c_{0}=\left(g_{m}+2 g_{q}\right) / 3$ and $c_{2}=$ $\left(g_{q}-g_{m}\right) / 3$. The term with $c_{0}$ is a central interaction, while the term with $c_{2}$ is spin-dependent.

When $g_{q} \approx g_{m}$ (e.g., for ${ }^{87} \mathrm{Rb}$ ), we have $c_{2}<<c_{0}$ and therefore the spin-dependent term is unimportant. In this case the single-mode approximation (SMA) works very well [3, 4, 5, 6, 12]. In SMA, a common mode function $\phi(\mathbf{r})$ is obtained by solving the Gross-Pitaevskii (GP) equation with the central interaction alone, while the small spin-dependent interaction is treated as a perturbation.

When $c_{2}$ is not very small, the perturbation treatment is poor. In this case, a three-mode approximation (TMA) is proposed. Instead of $\phi(\mathbf{r})$, three mode functions $\phi_{\mu}(\mathbf{r})$ are introduced, where $\mu=1,0$, and -1 are associated with the three components of spin. These functions obey a set of coupled GP equations with all the interactions taking into account 13 .

In the above theories the total spin $S$ of the condensate is not explicitly considered but only its Z-component $S_{Z}$. However, the interaction (eq.(11) assures that $S$ is exactly a good quantum number. In fact, the eigenstates should be $S$-dependent but $S_{Z}$-independent (if external magnetic field is not applied) . Therefore, a more perfect theory should preserve $S$. It is the aim of this paper to introduce a $S$-conserved description for spinor condensates. For this purpose, we need the knowledge from few-boson systems. This is discussed in the next section.

\section{2, Few-boson model systems}

Let us first inspect some few-boson 2-dimensional model systems with spin one and mass $m$, and they are confined by a harmonic trap with frequency $\omega$. When $\hbar \omega$ and $\sqrt{\frac{\hbar}{m \omega}}$ are used as units of energy and length, respectively, the Hamiltonian of the $N$-boson system reads

$$
H=\frac{1}{2} \sum_{i}\left(-\nabla_{i}^{2}+r_{i}^{2}\right)+\sum_{i<j} U_{i j}
$$

Using the method as given in the ref. 14, 15], the Hamiltonian for $N=2,3$, and 4 were diagonalized. Of course, both the orbital angular momentum $L$ and $S$ are good quantum numbers. However only the $L=0$ states are considered here. For a series of eigenstates with a given $S$, let the lowest one be called a first-state. Let the energy and wave function of a first-state be denoted as $E_{S}$ and $\Psi_{S}$. When $g_{q}+g_{m}=0.4$ is assumed, the evolution of $E_{S}$ in accord with $\beta=g_{q} / g_{m}$ is given in Fig. 1. In this figure, there are three groups of curves for the three cases $N=2,3$, and 4 , respectively. In each group the curves have distinct $S$ but the same $S_{Z}=0$. When $\beta=1$, the interaction is spin-independent and the ground state is degenerate against $S$. When $\beta$ deviates from 1, the interaction depends on the spin and, accordingly, the ground state splits into a band, the ground 
band. For an example, when $N=4$, the ground band contains three members with $S=0,2$, and 4 . When $\beta<1$, a pair of boson would be less repulsive if their spins are coupled to 2 instead of 0 (because $g_{q}<g_{m}$ ). Since in $\Psi_{4}$ any pair of bosons must couple to 2 , this state is lower than $\Psi_{2}$ and $\Psi_{0}$. On the contrary, when $\beta>1, \quad \Psi_{4}$ is the highest state of the band. Since the split of the ground state arises from the character of the interaction, it would remain when $N$ is large. In this case the spins will have many ways to couple with each other to form total spin-states with $S$ ranging from 0 (or 1) to $N$. It implies that, when $N$ is large, the ground band would contain many members distinct in their total spin-states. To clarify the structure of the ground band is another aim of this paper. Incidentally, since the members of the band are already orthogonal due to their spin structures, their spatial wave functions may be alike.

Let us analyze the wave functions to see the structure of the ground band of the few-boson systems. In the $S$-conserved form, the wave function is written as

$$
\Psi_{S}=\sum_{\lambda i} f_{S \lambda i} \theta_{S, \lambda i}
$$

where $\theta_{S, \lambda i}$ is the total spin-state with the good quantum number $S$, and it is also the $i-t h$ basis function of the $\lambda$ representation of the permutation group (e.g., refer to ref.[15]). $\quad f_{S \lambda i}$ is a function of spatial coordinates belonging to the same representation. The summation over $i$ is necessary to assure $\Psi_{S}$ to be totally symmetric. The summation over $\lambda$ responds to the possibility that some total spin-states distinct in $\lambda$ may have the same $S$ (e.g., for $N=3$ and $S=1$, there are three total spin-states $\theta_{1,\{3\} 1}, \theta_{1,\{21\} 1}$, and $\left.\theta_{1,\{21\} 2}\right)^{15}$. From eq.(3), for a $\Psi_{S}$, we can define the weight of a representation as

$$
W_{\lambda}=\sum_{i}<f_{S \lambda i} \mid f_{S \lambda i}>
$$

It turns out, from our numerical results, that all the states of the ground band are dominated by $\lambda=\{N\}$ component. E.g., when $N=4$, the second lowest state is $\Psi_{2}$ (cf. Fig.1). For $S=2$, there are six total spin-states belonging to the $\{4\},\{31\}$, and $\{22\}$ representations, respectively. When $\beta=0.6$, and $g_{m}+g_{q}$ varies in the range from 0 to 3 , our calculation shows that the $W_{\{4\}}$ of $\Psi_{2}$ ranges from 1 to 0.996 , it implies that the symmetry $\{4\}$ is dominant. The increase of $g_{m}+g_{q}$ causes only a very small decrease of $W_{\{4\}}$ from the unity. The symmetries other than $\{4\}$ are found to be important only in excited states but not in the ground band. This holds also when $\beta$ is given at other values. The underlying reason is that the $\lambda$ of the spatial wave function must be the same as the total spin-state. If $\lambda \neq\{N\}$, all the bosons can not condensate to the same spatial state, at least a boson should be spatially excited. This excitation will cause an explicit increase of energy. Since the ground band is an aggregation of the first-states, each of them will do their best to lower the energy. Therefore the $\{\mathrm{N}\}$-symmetry is pursued. Evidently, the above explanation remains valid not matter how large $N$ is. Thus, it is expected that the ground band of a large- $N$ system remains to be dominated by $\lambda=\{N\}$.

\section{3, Symmetrized total spin-states}

Let us now consider the ground band of the systems with many bosons . Based on the above discussion, only the case $\lambda=\{N\}$ is necessary to be considered. As a first step, we have to clarify the number of symmetrized total spin-states. For a $N$ - boson system, let $\theta_{S_{Z}}^{, j}$ denotes a normalized totally symmetric spin-state having only $S_{Z}$ conserved, where the superscript $j$ is introduced to number this kind of states. For a given $S_{Z}$, let the total number be denoted as $M_{Z}\left(S_{Z}\right)$, we have

$$
M_{Z}\left(S_{Z}\right)=\frac{1}{2}\left(N-S_{Z}-\frac{1-(-1)^{N-S_{Z}}}{2}\right)+1
$$

On the other hand, let $\theta_{S}^{k}$ denotes those having both $S$ and $S_{Z}$ conserved. For a given $S$, let the total number of $\theta_{S}^{k}$ be denoted as $M(S)$, which is related to $M_{Z}\left(S_{Z}\right)$ as

$$
M(S)=M_{Z}(S)-M_{Z}(S+1)=\left(1+(-1)^{N-S}\right) / 2
$$

From (6) we know that $M(S)$ is one if $N-S$ is even, or is zero if $N-S$ is odd. Therefore, the superscript $k$ is redundant, and a simpler notation $\theta_{S}$ is used hereafter. Evidently, $\theta_{S}$ is a simpler notation of $\theta_{S,\{N\} 1}$ defined before. For the calculation of matrix elements, the following formula is useful.

$$
\begin{aligned}
<\theta_{S}\left|\mathbf{F}_{1} \cdot \mathbf{F}_{2}\right| \theta_{S}> & =\frac{2}{N(N-1)}<\theta_{S}\left|\sum_{i<j} \mathbf{F}_{i} \cdot \mathbf{F}_{j}\right| \theta_{S}> \\
& =\frac{S(S+1)-2 N}{N(N-1)}
\end{aligned}
$$

Where the first equality holds because $\theta_{S}$ is totally symmetric, the second equality holds because

$$
2 \sum_{i<j} \mathbf{F}_{i} \cdot \mathbf{F}_{j}=\left(\sum_{i} \mathbf{F}_{i}\right)^{2}-\sum_{i} \mathbf{F}_{i}^{2}
$$

When $\theta_{S}$ are used for the spin-states, the spatial wave functions must be totally symmetric just as those for the scalar bosons. Therefore, in each member of the ground band, condensation would occur just as in the scalar system disregarding what $S$ is. Thus, as a reasonable approximation, $\Psi_{S}$ that has been given in eq. (3) is now simplified as

$$
\Psi_{S}=\Pi_{i=1}^{N} \phi_{S}\left(\mathbf{r}_{i}\right) \theta_{S}
$$

Where the subscript in $\phi_{S}$ responds to the fact that the spatial states may depend on $S$. Incidentally, the functions $\phi_{S}(\mathbf{r})$ with distinct $S$ are in general not mutually orthogonal. 


\section{4, The generalized Gross-Pitaevskii equation}

Let us insert (9) into the three-dimensional Schrödinger equation

$$
H \Psi_{S}=E_{S} \Psi_{S}
$$

where $H$ is given in (2). Using the standard variational procedure, a generalized Gross-Pitaevskii (GP) equation can be derived as

$$
\left[\frac{1}{2}\left(-\nabla^{2}+r^{2}\right)+(N-1) g_{S} \rho_{S}\right] \phi_{S}(r)=\varepsilon_{S} \phi_{S}(r)
$$

where

$$
\begin{aligned}
g_{S} & =c_{0}+<\theta_{S}\left|\mathbf{F}_{1} \cdot \mathbf{F}_{2}\right| \theta_{S}>c_{2} \\
& =c_{0}+\frac{S(S+1)-2 N}{N(N-1)} c_{2}
\end{aligned}
$$

$\phi_{S}(r)$ is normalized and isotropic, $\rho_{S}=\phi_{S}(r)^{*} \phi_{S}(r)$ and $\varepsilon_{S}$ is related to the eigenenergy $E_{S}$ as

$$
E_{S}=N \varepsilon_{S}-\frac{N(N-1)}{2} g_{S} \overline{\rho_{S}}
$$

where

$$
\overline{\rho_{S}}=\int d \mathbf{r} \phi_{S}(r)^{*} \rho_{S} \phi_{S}(r)
$$

The generalized GP equation is the same as the usual one with only one exception, namely the strength $g_{S}$ is not a constant but $S-$ and $N$-dependent. Consequently, the bosons in each state of the ground band condensate into a specific single-particle states $\phi_{S}$ depending on $S$. From (6) we know that the band contains totally $N / 2+1$ states if $N$ is even with their $S$ ranging from $0,2,4$, to $N$, or contains $(N+1) / 2$ states if $N$ is odd with $S$ ranging from $1,3,5$, to $N$. When $\beta=1$, the generalized GP equation will automatically reduce to the usual GP equation. When $\beta>1, c_{2}$ is positive, a larger $S$ will lead to a larger $g_{S}$, and therefore a higher level. Thus, the upmost level of the ground band has $S=N$, while the ground state has $S=0$. On the contrary, when $\beta<1$, the ground state has $S=N$. This finding coincides with previous findings [4].

Let us go to realistic cases (three-dimensional) with a large $\mathrm{N}$ to inspect the structure of the ground band. For ${ }^{23} \mathrm{Na}$ atoms, we have $g_{m}=6.351 \times 10^{-4} \sqrt{\omega}$ and $\beta=1.10$ . For ${ }^{87} \mathrm{Rb}$ atoms, we have $g_{m}=2.52 \times 10^{-3} \sqrt{\omega}$ and $\beta=0.986$. By solving (11), the ground state energies $E_{g}$ per particle with respect to $N \omega^{1 / 2}$ are plotted in Fig. 2 . In order to see the broadening of the ground band, $\left(E_{S}-E_{S_{m i n}}\right) / N$ with respect to $S / N$ is plotted in Fig. 3 , where $\omega=100 \mathrm{~Hz}$ is given, the subscript $S_{\text {min }}=0$ (if $N$ even), or $=1$ (if $N$ odd).

Now, let us evaluate the level-density. When $S / N<<$ 1 , eq. (11) can be solved perturbatively (the perturbation is caused by $\left.g_{S}-g_{S_{m i n}}\right)$, we obtain

$\varepsilon_{S}-\varepsilon_{S_{\min }}=(N-1)\left(g_{S}-g_{S_{m i n}}\right) \bar{\rho}_{S_{\min }}=c_{2} \frac{S(S+1)}{N} \bar{\rho}_{S_{\min }}$

Thereby the difference $E_{S}-E_{S_{\min }}$ can be obtained, and we have the level-density

$$
\begin{aligned}
D\left(E_{S}\right) & =\left|\frac{d n}{d E_{S}}\right|=1 /\left(\left|c_{2}\right| \bar{\rho}_{S_{\text {min }}}(2 S+1)\right) \\
& =S(S+1) /\left(2\left|E_{S}-E_{S_{\text {min }}}\right|(2 S+1)\right)
\end{aligned}
$$

where $d n$ is the number of levels in $d E_{S}$. Since $\left|E_{S}-E_{S_{\min }}\right|$ is a very small quantity in the vicinity of $E_{S_{\min }}$, the levels are extremely dense in the vicinity of the ground state (if $\beta>1$ ) or in the top of the ground band (if $\beta<1$ ). This is an interesting point.

When $S / N$ is not very small, via a numerical evaluation with a high precision, $E_{S}$ can be represented by a parabolic curve

$$
E_{S}=E_{S_{\min }} \pm N\left(\frac{S}{N}\right)^{2} d
$$

where $d$ is a function of $N$ as given in the inset of Fig. 3 , the positive (negative) sign is for $\beta>1(<1)$. The band width $E_{b w}=\left|E_{N}-E_{S_{m i n}}\right|$ is given in Fig. 4 versus $N \omega^{1 / 2}$. The level-density is then

$$
D\left(E_{S}\right)=\frac{1}{4} \sqrt{\frac{N}{\left|E_{S}-E_{S_{\min }}\right| d}}
$$

They are given in Fig. 5, where $\omega=100 \mathrm{~Hz}$ is assumed.

If the generalized GP equation is not solved exactly but perturbatively (the perturbation is caused by $g_{S}-c_{0}$ ), then the resultant energies are identical to those given by the $\mathrm{SMA}^{4}$.

\section{5 , Final remarks}

In conclusion, we have derived a generalized GP equation based on a $S$-conserved formalism . This equation can describe not only the ground state but the whole ground band. In the band the states having larger $S$ are higher (lower) in $E_{S}$ if $\beta>(<) 1$. It is recalled that $S$ is in fact a good quantum number. The present theoretical framework satisfies this requirement, and has a very simple formalism to facilitate numerical calculations. Furthermore, (9) is the only approximation, which is comparable to that used for deriving the well known GP equation. Therefore, the reliabilities of these two equations are comparable. Thus, this work is an improvement of the SMA and TMA, while at the same time avoids perturbative treatment or the difficulty of solving coupled equations.

Acknowledgment: We appreciate the support by NSFC under the grants 90103028 and 90306016 . 
[1] D. M. Stamper-Kurn, M. R. Andrews, A. P. Chikkatur, S. Inouye, H. J. Miesner, J. Stenger, and W. Ketterle, Phys. Rev. Lett. 80, 2027 (1998)

[2] J. I. Cirac, M. Lewenstein, K. Molmer, and P. Zoller, Phys. Rev. A 57, 1208 (1998)

[3] L. M. Duan, J. I. Cirac, and P. Zoller, Phys. Rev. A 65, 033619 (2002)

[4] C. K. Law, H. Pu, and N. P. Bigelow, Phys. Rev. Lett. 81, 5257 (1998)

[5] E. Goldstein and P. Meystre, Phys. Rev. A 59, 3896 (1998)

[6] T. L. Ho and S. K. Yip, Phys. Rev. Lett. 84, 4031 (2000)

[7] T. L. Ho, Phys. Rev. Lett. 81, 742 (1998)

[8] T. Ohmi and K. Machida, J. Phys. Soc. Japan, 67, 1822 (1998)

[9] W. Zhang and D.F. Walls, Phys. Rev. A 57, 1248 (1998)

[10] E.G.M. van Kempen et al, Phys. Rev. Lett. 88, 093201 (2002)

[11] A. Crubelier, et al, Eur. Phys. J. D 6, 211 (1999)

[12] M. Koashi, and M. Ueda, Phys. Rev. Lett. 84, 1066 (2000)

[13] S. Yi, Ö. E. Müstecaphoğlu, C. P. Sun, and L. You, Phys. Rev. A 66, 011601R (2002)

[14] C. G. Bao, T. Y. Shi, Phys. Rev. A 68, 032509 (2003)

[15] C. G. Bao, T. Y. Shi, Phys. Rev. A 66, 013613 (2002) 


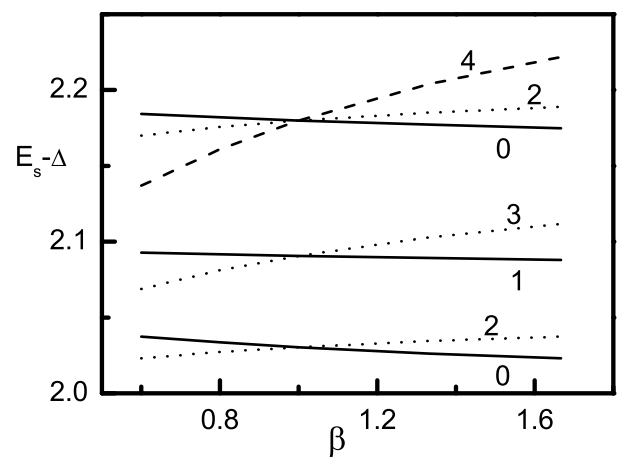

FIG. 1: The evolution of $E_{S}$ (in $\hbar \omega$ ) with $\beta$ in few -boson systems. Where the lowest two curves, the two in the middle, and the upper three are for 2-, 3- and 4-boson systems, respectively. $S$ is marked by the Curves. $\Delta$ is introduced to shift the curves, $\Delta=0,1$, and 2 for $N=2,3$, and 4 systems, respectively.

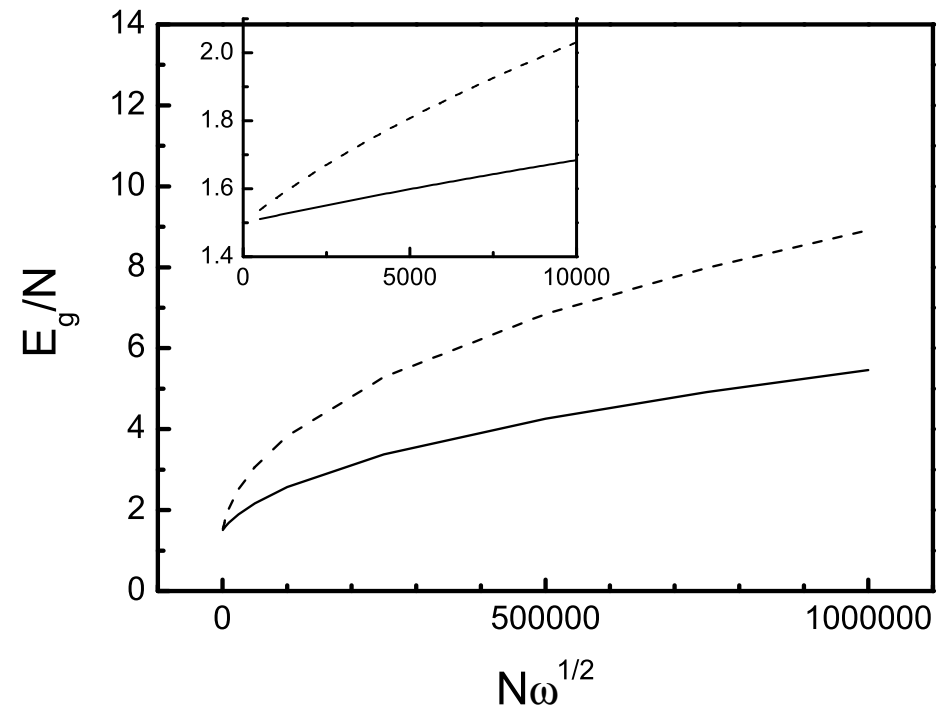

FIG. 2: The ground state energy $E_{g}$ per particle (in $\hbar \omega$ ) versus $N \omega^{1 / 2}$. The total spin of the ground state is 0 or 1 (if ${ }^{23} \mathrm{Na}$ ), or is $N$ (if ${ }^{87} \mathrm{Rb}$ ). In Fig. 2 to 5 the solid (dashed) lines are for ${ }^{23} \mathrm{Na}\left({ }^{87} \mathrm{Rb}\right)$. The inset is just for smaller $N \omega^{1 / 2}$ 


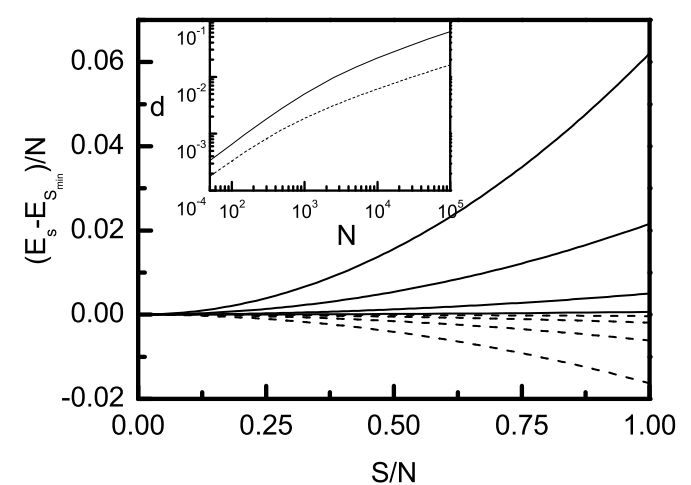

FIG. 3: $\left(E_{S}-E_{S_{\min }}\right) / N($ in $\hbar \omega)$ versus $S / N$ with $\omega=100 H z$ - The four solid (dashed) lines from the lowest to highest (highest to lowest) are for $N=10^{2}, 10^{3}, 10^{4}$, and $10^{5}$, respectively. The inset is $d$ versus $N$.

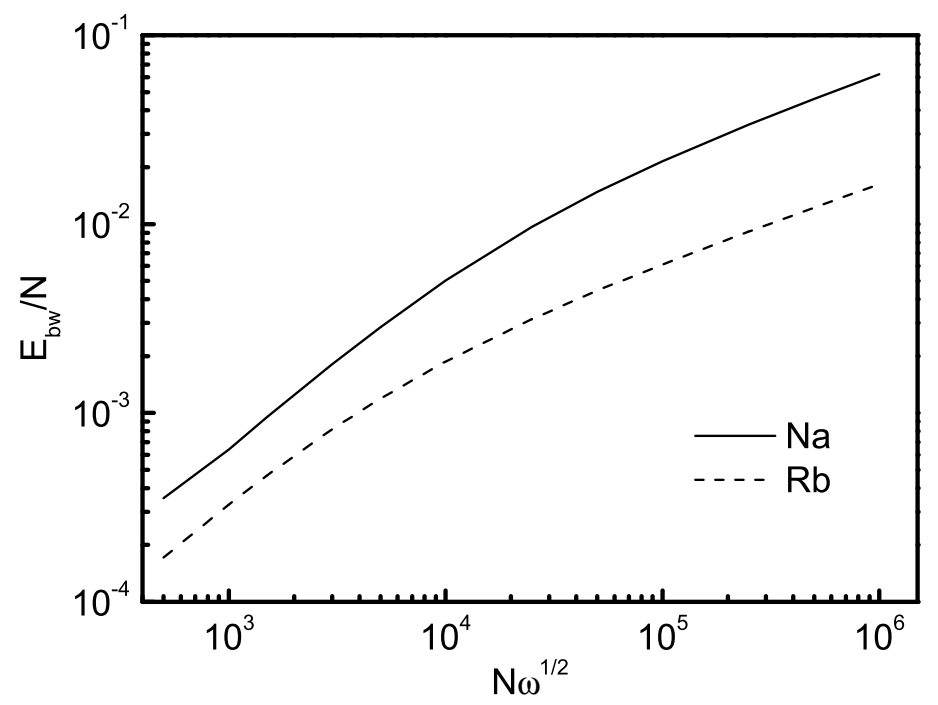

FIG. 4: The band width $E_{b w} / N$ (in $\left.\hbar \omega\right)$ versus $N \omega^{1 / 2}$. 


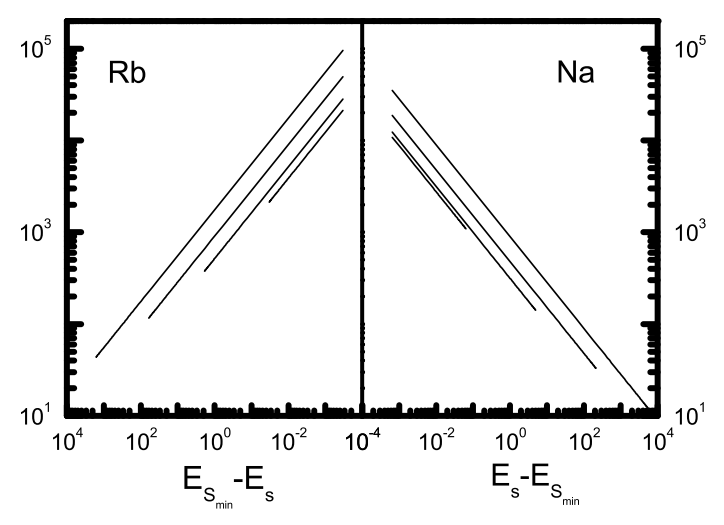

FIG. 5: The level-density $D\left(E_{S}\right)$ versus $E_{S}-E_{S_{\min }}$ (in $\hbar \omega$ ) with $\omega=100 \mathrm{~Hz}$. The lines from the shortest to longest are for $N=10^{2}, 10^{3}, 10^{4}$, and $10^{5}$, respectively. 